\title{
Secondary Atmospheres on HD 219134 b and c
}

\author{
Caroline Dorn ${ }^{1}$ and Kevin Heng ${ }^{2,3,4}$ (DD \\ ${ }^{1}$ University of Zurich, Institut of Computational Sciences, University of Zurich, Winterthurerstraße 190, CH-8057, Zurich, Switzerland; cdorn@physik.uzh.ch \\ ${ }^{2}$ University of Bern, Center for Space and Habitability, Gesellschaftsstraße 6, CH-3012, Bern, Switzerland; kevin.heng@csh.unibe.ch \\ 3 Johns Hopkins University, Department of Earth and Planetary Sciences, 301 Olin Hall, Baltimore, MD 21218, USA \\ ${ }^{4}$ Johns Hopkins University, Department of Physics and Astronomy, Bloomberg Center for Physics and Astronomy, Baltimore, MD 21218, USA \\ Received 2017 October 13; revised 2017 November 20; accepted 2017 November 20; published 2018 January 23
}

\begin{abstract}
We analyze the interiors of HD $219134 \mathrm{~b}$ and c, which are among the coolest super-Earths detected thus far. Without using spectroscopic measurements, we aim at constraining if the possible atmospheres are hydrogen-rich or hydrogen-poor. In the first step, we employ a full probabilistic Bayesian inference analysis to rigorously quantify the degeneracy of interior parameters given the data of mass, radius, refractory element abundances, semimajor axes, and stellar irradiation. We obtain constraints on structure and composition for core, mantle, ice layer, and atmosphere. In the second step, we aim to draw conclusions on the nature of possible atmospheres by considering atmospheric escape. Specifically, we compare the actual possible atmospheres to a threshold thickness above which a primordial $\left(\mathrm{H}_{2}\right.$-dominated) atmosphere can be retained against evaporation over the planet's lifetime. The best-constrained parameters are the individual layer thicknesses. The maximum radius fraction of possible atmospheres are 0.18 and $0.13 R$ (radius), for planets $\mathrm{b}$ and $\mathrm{c}$, respectively. These values are significantly smaller than the threshold thicknesses of primordial atmospheres: 0.28 and $0.19 R$, respectively. Thus, the possible atmospheres of planets $\mathrm{b}$ and $\mathrm{c}$ are unlikely to be $\mathrm{H}_{2}$-dominated. However, whether possible volatile layers are made of gas or liquid/solid water cannot be uniquely determined. Our main conclusions are (1) the possible atmospheres for planets $\mathrm{b}$ and $\mathrm{c}$ are enriched and thus possibly secondary in nature, and (2) both planets may contain a gas layer, whereas the layer of HD 219134 b must be larger. HD 219134 c can be rocky.
\end{abstract}

Key words: planets and satellites: atmospheres - planets and satellites: composition - planets and satellites: individual (HD 219134 b, HD 219134 c) - planets and satellites: interiors - planets and satellites: terrestrial planets

\section{Introduction}

\subsection{Motivation}

Little is known about compositional and structural diversity of super-Earths. We often consider super-Earths to be distinct from sub-Neptunes in terms of their volatile fraction. In fact, there is an intriguing transition around $1.5 \mathrm{R}_{\oplus}$, above which most planets appear to contain a significant amount of volatiles (e.g., Dressing \& Charbonneau 2015; Rogers 2015). The distribution of planet densities and radii suggest a transition that is continuous rather than stepwise (Leconte et al. 2015), although the limited number of available observations might not allow a firm conclusion yet (Rogers 2015).

A key criterion to distinguish super-Earths from subNeptunes is the origin of its atmosphere. Super-Earths' atmospheres are thought to be dominated by outgassing from the interior, whereas sub-Neptunes have accreted and retained a substantial amount of primordial hydrogen and helium. The atmospheric scale height will be significantly larger in the latter case since it scales as the reciprocal of the mean molecular mass. Consequently, the radius fraction of volatiles is often used to distinguish between super-Earths and sub-Neptunes.

The nature of an atmosphere, be it primordial or secondary, helps to clearly categorize a planet. Atmospheres can have three different origins: (1) accreted nebular gas from the protoplanetary disk (primordial origin), (2) gas-release during disruption of accreting volatile-enriched planetesimals, or (3) outgassing from the interior (secondary origin). The timescales associated with (1-2) and (3) are very different. An atmosphere that is dominated by outgassed planetesimal disruption (2) can theoretically be significantly different from a hydrogen-helium atmosphere (e.g., Hashimoto et al. 2007; Schaefer \& Fegley 2007; Elkins-Tanton \& Seager 2008; Zahnle et al. 2010; Fortney et al. 2013; Venturini et al. 2016). Venturini et al. (2016) show that enriched gas layers speed up the accretion of gas from the primordial disk, which explains large fractions of $\mathrm{H} / \mathrm{He}$ for intermediate mass planets. However, to what extent atmospheres of low-mass planets can be enriched (e.g., in water) and sustain their metallicity over their lifetime is subject of ongoing research. For the closein super-Earths HD $219134 \mathrm{~b}$ and c, we consider the scenarios (1) and (3). In other words, we use the term primordial to refer to $\mathrm{H}_{2}$-dominated atmospheres that are pristine and compositionally unaffected by subsequent physical or chemical processing including atmospheric escape (e.g., Lammer et al. 2013; Hu et al. 2015) or interaction with the rocky interior.

The atmospheres of close-in planets are subject to significant mass loss (atmospheric escape), driven by extreme ultraviolet and X-ray heating from their stars. The goal of this study is to present a method for determining if a planet may host a gaseous layer, and if this gas layer is hydrogen-dominated (primordial) or dominated by high mean molecular masses (secondary). Our method is different and complementary to studies that use spectroscopic signatures to distinguish between hydrogen-rich and hydrogen-poor atmospheres (e.g., Miller-Ricci et al. 2009). We focus on the HD 219134 system, which hosts multiple planets. Two of which fall in the super-Earth regime. Both planets b and c are transiting (Vogt et al. 2015; Gillon et al. 2017) and represent together the coolest super-Earth pair yet detected in a star system (Figure 1).

The characterization of two planets from the same system benefits from possible compositional correlations between 


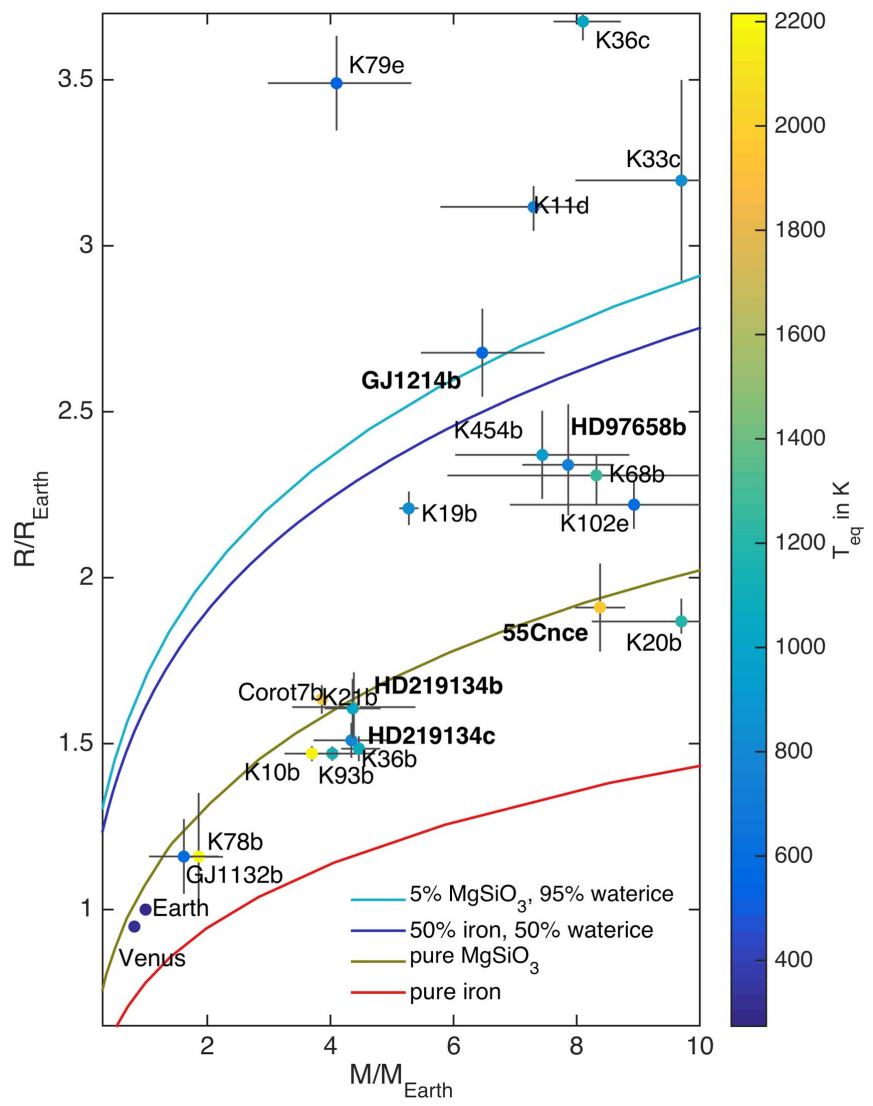

Figure 1. Mass-radius diagram for planets below $2.7 \mathrm{R}_{\oplus}$ and $10 \mathrm{M}_{\oplus}$ and mass uncertainties better than $20 \%$ in general. HD $219134 \mathrm{~b}$ and c are among the coolest exoplanets yet detected regarding their equilibrium temperature (in color). Planets in boldface are included in the comparative study in Section 3.3.

them. We can expect a correlation in relative abundance of refractory elements (Sotin et al. 2007). Abundances measured in the photosphere of the host star can be used as proxies for the relative bulk abundances, namely $\mathrm{Fe} / \mathrm{Si}$ and $\mathrm{Mg} / \mathrm{Si}$ (Dorn et al. 2015). Here, we use different photospheric measurements on HD 219134, compiled by Hinkel et al. (2014). These bulk abundance constraints in addition to mass, radius, and stellar irradiation are the data that we use to infer structure and composition of the planets.

A rigorous interior characterization that accounts for data and model uncertainty can be done sensibly using Bayesian inference analysis, for which we use the generalized method of Dorn et al. (2016b). The previous work of Dorn et al. (2015, 2016b) showed that Bayesian inference analysis is a robust method for quantifying interior parameter degeneracy for a given (observed) exoplanet. While Dorn et al. (2015) focused on purely rocky planets, a generalized method for super-Earths and mini-Neptunes was developed by Dorn et al. (2016b) by including volatiles (liquid and high-pressure ices, and gas layers). Inferred confidence regions of interior parameters are generally large, which emphasizes the need to utilize extra data that further informs one about a planet's composition and structure. Here, we investigate additional considerations on atmospheric escape to further constrain the nature of the atmosphere.

Similar to the previous works, we assume that planets that are made of distinct layers, i.e., an iron core, a silicate mantle, a water layer, and a gas layer as illustrated in Figure 2. The use of an inference analysis allows us to account for the degeneracy

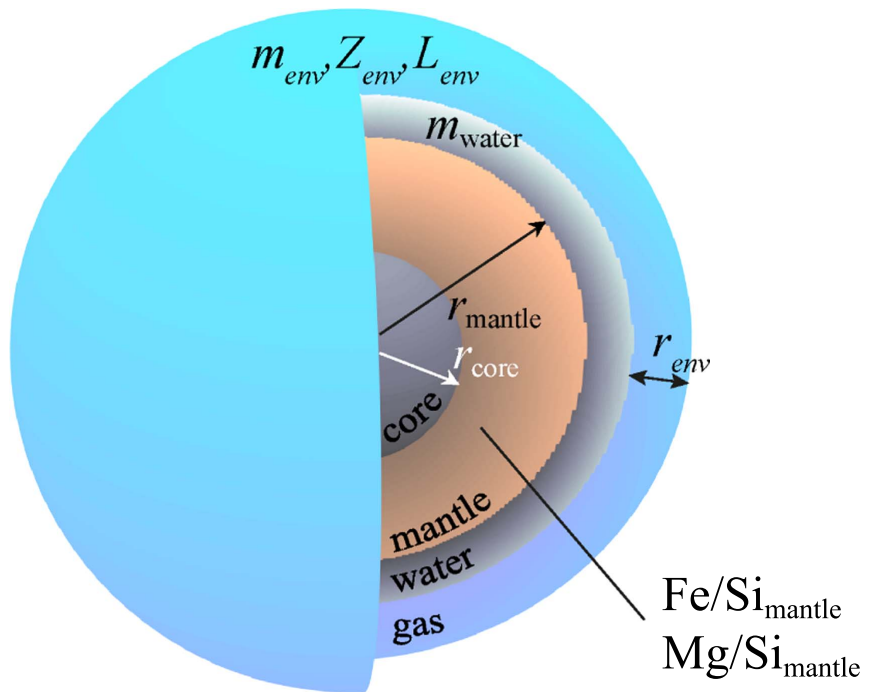

Figure 2. Illustration of interior parameters: core size $\left(r_{\text {core }}\right)$, mantle composition $\left(\mathrm{Fe} / \mathrm{Si}_{\text {mantle }}, \mathrm{Mg} / \mathrm{Si}_{\text {mantle }}\right)$, mantle size $\left(r_{\text {mantle }}\right)$, water mass fraction $\left(m_{\text {water }}\right)$, intrinsic luminosity $\left(L_{\text {env }}\right)$, gas mass $\left(m_{\text {env }}\right)$, gas metallicity $\left(Z_{\text {env }}\right)$, and atmospheric thickness $\left(r_{\text {env }}\right)$.

among the layer properties, i.e., core size; mantle size and composition; water mass fraction; gas mass fraction and metallicity; and intrinsic luminosity. In this study, we account for interior degeneracy and calculate the robust confidence regions of atmospheric thicknesses $\left(r_{\text {env }}\right)$. These inferred thicknesses $r_{\text {env }}$ are then compared with theoretically possible thicknesses of a $\mathrm{H}_{2}$-dominated atmosphere. The theoretically possible range of a $\mathrm{H}_{2}$-dominated atmospheres is restricted due to atmospheric escape, i.e., too thin $\mathrm{H}_{2}$-dominated atmospheres cannot be retained over a planet's lifetime. This implies a threshold thickness below which $\mathrm{H}_{2}$-dominated atmospheres cannot be retained. Here, we present how this threshold thickness $(\Delta R)$ can be estimated. The comparison between $\Delta R$ and $r_{\text {env }}$ is a key aspect of our study and allows us to draw conclusions about the nature of possible planetary atmospheres.

\subsection{Concept and Method}

We first describe the method conceptually before providing the technical details later in the paper. Consider a planet orbiting close to its star, which emits ultraviolet and X-ray radiation. Planet formation occurs on short timescales $\left(\sim 10^{6}-10^{8}\right.$ years) and is essentially instantaneous over the lifetime of a $\sim 1-10$ Gyr-old star. Immediately after the planet has formed, it retains a hydrogen-dominated atmosphere, which is then continuously eroded until the present time. We take the total time lapsed to be the age of the star $\left(t_{\star}\right)$.

The total mass of lost primordial atmosphere, $M_{\text {env,lost }}(t)$, increases over time due to atmospheric escape. Over the lifetime $t_{\star}$, the total escaped mass is $M_{\text {env,lost }}\left(t_{\star}\right)$, which we convert to a fraction of the planetary radius, $\Delta R / R$. Atmospheric escape can erode $\Delta R$ worth of atmosphere over the age of the star. Independent of $\Delta R$ and from our Bayesian inference analysis, we can estimate the possible range of atmospheric thicknesses at the present time, $r_{\text {env }}\left(t_{\star}\right)$. If $r_{\text {env }}\left(t_{\star}\right)<\Delta R$, then the atmosphere is not $\mathrm{H}_{2}$-dominated because any $\mathrm{H}_{2}$ atmosphere would have been eroded away. Thus, $\Delta R$ may be visualized as the threshold thickness above which a primordial atmosphere can be retained against atmospheric escape over a 
time $t_{\star}$. The comparison between $r_{\text {env }}\left(t_{\star}\right)$ and $\Delta R$ is a key aspect of this study.

The outline of this study is as follows. We first discuss the method of characterizing planet interiors. We explain how we approximate the amount of primordial atmosphere that may be lost due to stellar irradiation and how we relate this to a threshold thickness of a primordial atmosphere. On the basis of these estimates, we demonstrate how we infer the atmospheric origin. We show results for HD $219134 \mathrm{~b}$ and c, and compare them with $55 \mathrm{Cnc}$ e, HD 97658 b, and GJ 1214 b. In an attempt to get an idea of the distribution of enriched (secondary) atmospheres, we apply the method to low-mass planets $\left(<10 \quad \mathbf{M}_{\oplus}\right)$. We finish with a discussion and conclusions.

Note that we use the terms atmosphere and gas layer synonymously. The atmosphere/gas layer model comprises a radiative layer on top of a convection-dominated envelope.

\section{Methodology}

\subsection{Interior Characterization}

Using the generalized Bayesian inference analysis of Dorn et al. (2016b), which employs a Markov chain Monte Carlo (McMC) method, we rigorously quantify the degeneracy of the following interior parameters for a general planet:

1. Core: core size $\left(r_{\text {core }}\right)$;

2. Mantle: mantle composition $\left(\mathrm{Fe} / \mathrm{Si}_{\text {mantle }}, \mathrm{Mg} / \mathrm{Si}_{\text {mantle }}\right)$ and size $\left(r_{\text {mantle }}\right)$;

3. Water: water mass fraction ( $\left.m_{\text {water }}\right)$; and

4. Gas: intrinsic luminosity $\left(L_{\mathrm{env}}\right)$, gas mass $\left(m_{\mathrm{env}}\right)$, and metallicity $\left(Z_{\text {env }}\right)$.

From the posterior distribution of those interior parameters, we can compute the posterior distribution of the thickness of a possible gas layer $\left(r_{\mathrm{env}}\right)$, which we then use to infer if the gas layer is hydrogen-rich or poor (Section 2.3). Regarding the volatile-rich layers, our parameterization allows us to produce planet structures that range from (1) purely rocky to (2) thick water layers with no additional gas layer to (3) thick gas layers without water layers below. The latter structure (3) determines the largest values of $r_{\text {env }}$. Figure 2 illustrates the interior parameters of interest.

The considered data comprise

1. mass, $M$,

2. radius, $R$,

3. bulk abundance constraints on $\mathrm{Fe} / \mathrm{Si}_{\text {bulk }}$ and $\mathrm{Mg} / \mathrm{Si}_{\text {bulk, }}$, and minor elements $\mathrm{Na}, \mathrm{Ca}, \mathrm{Al}$,

4. semimajor axes, $a$,

5. stellar irradiation (namely, effective temperature $T_{\text {eff }}$ and stellar radius $\left.R_{\star}\right)$.

For $\mathrm{Fe} / \mathrm{Si}_{\text {bulk }}, \mathrm{Mg} / \mathrm{Si}_{\text {bulk }}$ and minor elements, we use their equivalent stellar ratios as proxies that can be measured in the stellar photosphere (Dorn et al. 2015).

The prior distributions of the interior parameters are listed in Table 1. The priors are chosen conservatively. The cubic uniform priors on $r_{\text {core }}$ and $r_{\text {mantle }}$ reflect equal weighing of masses for both core and mantle. Prior bounds on $\mathrm{Fe} / \mathrm{Si}_{\text {mantle }}$ and $\mathrm{Mg} / \mathrm{Si}_{\text {mantle }}$ are determined by the host star's photospheric abundance proxies. As iron is distributed between core and mantle, $\mathrm{Fe} / \mathrm{Si}_{\text {bulk }}$ only sets an upper bound on $\mathrm{Fe} / \mathrm{Si}_{\text {mantle }} \mathrm{A}$ $\log$-uniform prior is set for $m_{\mathrm{env}}$ and $L_{\mathrm{env}}$. A uniform prior in
Table 1

Prior Ranges

\begin{tabular}{lll}
\hline \hline Parameter & \multicolumn{1}{c}{ Prior Range } & Distribution \\
\hline$r_{\text {core }}$ & $(0.01-1) r_{\text {mantle }}$ & Uniform in $r_{\text {core }}^{3}$ \\
$\mathrm{Fe} / \mathrm{Si}_{\text {mantle }}$ & $0-\mathrm{Fe} / \mathrm{Si}_{\text {star }}$ & Uniform \\
$\mathrm{Mg} / \mathrm{Si}_{\text {mantle }}$ & $\mathrm{Mg} / \mathrm{Si}_{\text {star }}$ & Gaussian \\
$r_{\text {mantle }}$ & $(0.01-1) R$ & Uniform in $r_{\text {mantle }}^{3}$ \\
$m_{\text {water }}$ & $0-0.98 M$ & Uniform \\
$m_{\text {env }}$ & $0-m_{\text {env,max }}$ & Uniform in log-scale \\
$L_{\text {env }}$ & $10^{18}-10^{23} \mathrm{erg} \mathrm{s}^{-1}$ & Uniform in log-scale \\
$Z_{\text {env }}$ & $0-1$ & Uniform \\
\hline
\end{tabular}

$Z_{\text {env }}$ equally favors metal-poor and metal-rich atmospheres, which seems appropriate for secondary atmospheres. In Section 3.1, we investigate the effect of different priors on $Z_{\text {env }}$.

In this study, the planetary interior is assumed to be composed of a pure iron core; a silicate mantle comprising the oxides $\mathrm{Na}_{2} \mathrm{O}-\mathrm{CaO}-\mathrm{FeO}-\mathrm{MgO}-\mathrm{Al}_{2} \mathrm{O}_{3}-\mathrm{SiO}_{2}$; pure water layer; and an atmosphere of $\mathrm{H}, \mathrm{He}, \mathrm{C}$, and $\mathrm{O}$.

The structural model for the interior uses self-consistent thermodynamics for core, mantle, high-pressure ice, and water ocean, and to some extent also atmosphere. For the core density profile, we use the equation of state (EoS) fit of iron in the hcp (hexagonal close-packed) structure provided by Bouchet et al. (2013) on ab initio molecular dynamics simulations. We assume a solid-state iron, as the density increase due to solidification in the Earth's core is small $\left(0.4 \mathrm{~g} \mathrm{~cm}^{-3}\right.$, or $\left.3 \%\right)$ (Dziewonski \& Anderson 1981). For the silicate mantle, we compute equilibrium mineralogy and density as a function of pressure, temperature, and bulk composition by minimizing Gibbs free energy (Connolly 2009). For the water layers, we follow Vazan et al. (2013) using a quotidian EoS and above 44.3 GPa, we use the tabulated EoS from Seager et al. (2007) that is derived from DFT simulations. Depending on pressure and temperature, the water can be in solid, liquid, or vapor phase. We assume an adiabatic temperature profile within core, mantle, and water layers. The surface temperature of the water layer is set to the equilibrium temperature of the planet.

For the gas layer, we solve the equations of hydrostatic equilibrium, mass conservation, and energy transport. For the EoS of elemental compositions of $\mathrm{H}, \mathrm{He}, \mathrm{C}$, and $\mathrm{O}$, we employ the Chemical Equilibrium with Applications (CEA) package (Gordon \& McBride 1994), which performs chemical equilibrium calculations for an arbitrary gaseous mixture, including dissociation and ionization and assuming ideal gas behavior. The metallicity $Z_{\text {env }}$ is the mass fraction of $\mathrm{C}$ and $\mathrm{O}$ in the gas layer, which can range from 0 to 1 . For the gas layer, we assume an irradiated layer on top of a convective-dominated envelope, for which we assume a semi-gray, analytic, global temperature averaged profile (Guillot 2010; Heng et al. 2014). The boundary between the irradiated layer and the underlying envelope is defined where the optical depth in visible wavelength is $100 / \sqrt{3}$ (Jin et al. 2014). Within the envelope, the usual Schwarzschild criterion is used to distinguish between convective and radiative layers. The planet radius is defined where the chord optical depth becomes 0.56 (Lecavelier des Etangs et al. 2008).

We refer to model I in Dorn et al. (2016b) for more details on both the inference analysis and the structural model. 
Table 2

Summary of Planetary Data (Motalebi et al. 2015; Gillon et al. 2017)

\begin{tabular}{lcc}
\hline \hline Parameter & HD 219134 b & HD 219134 c \\
\hline$R / \mathrm{R}_{\oplus}$ & $1.606 \pm 0.086$ & $1.515 \pm 0.047$ \\
$M / \mathrm{M}_{\oplus}$ & $4.36 \pm 0.44$ & $4.34 \pm 0.22$ \\
$g_{\text {surf }}\left[\mathrm{cm} / \mathrm{s}^{-2}\right]$ & 1656 & 1865 \\
$T_{\text {eq }}[\mathrm{K}]$ & 1025 & 784 \\
$a[\mathrm{au}]$ & 0.038 & 0.065 \\
\hline
\end{tabular}

\subsection{Estimating the Threshold Thickness $\Delta R$ of a Primordial Atmosphere Layer Considering Atmospheric Escape}

We approximate $\Delta R$ by the atmospheric layer thickness that corresponds to the accumulated mass of hydrogen that may be lost over the planet's lifetime $\left(M_{\text {env,lost }}\right)$. Loss rates are determined by X-ray irradiation from the star and mass-loss efficiencies. Hydrostatic balance is used to calculate the layer thickness $\Delta R$ corresponding to a primordial atmosphere of mass $M_{\text {env,lost }}$ The detailed calculation of $\Delta R$ involves several steps that are discussed in the following.

Let the layer thickness $\Delta R$ be the difference in radius attributed to a primordial atmosphere. If we assume this layer to be in hydrostatic equilibrium, then this difference in radius is

$$
\Delta R=H \ln \left(\frac{P_{\mathrm{b}}}{P_{\mathrm{t}}}\right),
$$

where $H$ is pressure scale height, and $P_{\mathrm{b}}$ is the pressure at the bottom of the layer, which we will derive in the next paragraphs. $P_{\mathrm{t}}$ is the pressure at the top of the layer, corresponding to the transit radius (Heng 2016)

$$
P_{\mathrm{t}} \approx \frac{g}{\kappa} \sqrt{\frac{H}{2 \pi R}} .
$$

If we assume a mean opacity of $\kappa=0.1 \mathrm{~cm}^{2} \mathrm{~g}^{-1}$ (Freedman et al. 2014), then for both the $\mathrm{b}$ and c planets we get $P_{\mathrm{t}} \approx 1 \mathrm{mbar}$.

The pressure scale height, $H$, is calculated assuming a hydrogen-dominated layer (mean molecular mass $\mu=2 \mathrm{~g} \mathrm{~mol}^{-1}$ ) and using the equilibrium temperature $T_{\mathrm{eq}}$,

$$
H=\frac{T_{\mathrm{eq}} R^{*}}{g_{\text {surf }} \mu},
$$

where $g_{\text {surf }}$ is surface gravity, and $R^{*}$ is the universal gas constant $\left(8.3144598 \mathrm{~J} \mathrm{~mol}^{-1} \mathrm{~K}^{-1}\right)$. The estimates in Heng (2016) suggest that the assumption of $T=T_{\mathrm{eq}}$ is reasonable. Values for $g_{\text {surf }}$ and $T_{\text {eq }}$ are listed in Tables 2 and 3.

The pressure at the bottom of the layer $P_{\mathrm{b}}$ corresponds to the accumulated mass of escaped hydrogen over the planet's lifetime $M_{\text {env,lost }}$,

$$
P_{\mathrm{b}}=\frac{g M_{\mathrm{env}, \text { lost }}}{4 \pi R^{2}},
$$

which is simply a restatement of Newton's second law. $M_{\text {env,lost }}$ is approximated by atmospheric escape considerations. Dimensional analysis yields an expression for the atmospheric escape rate

$$
\dot{M}=\frac{\pi \eta F_{\mathrm{X}} R^{2}}{E_{g}},
$$

Table 3

Summary of Stellar Data (Motalebi et al. 2015)

\begin{tabular}{ll}
\hline \hline Parameter & \multicolumn{1}{c}{ HD 219134} \\
\hline$R_{\text {star }} / R_{\text {Sun }}$ & $0.778 \pm 0.005$ \\
$T_{\text {eff }}$ in K & $4699 \pm 16$ \\
{$[\mathrm{Fe} / \mathrm{H}]$} & $0.04-0.84$ \\
{$[\mathrm{Fe} / \mathrm{H}]_{\text {median }}$} & 0.13 \\
{$[\mathrm{Mg} / \mathrm{H}]$} & $0.09-0.37$ \\
{$[\mathrm{Mg} / \mathrm{H}]_{\text {median }}$} & 0.32 \\
{$[\mathrm{Si} / \mathrm{H}]$} & $0.04-0.27$ \\
{$[\mathrm{Si} / \mathrm{H}]_{\text {median }}$} & 0.12 \\
{$[\mathrm{Na} / \mathrm{H}]$} & $0.17-0.32$ \\
{$[\mathrm{Na} / \mathrm{H}]_{\text {median }}$} & 0.19 \\
{$[\mathrm{Al} / \mathrm{H}]$} & $0.16-0.29$ \\
{$[\mathrm{Al} / \mathrm{H}]_{\text {median }}$} & 0.23 \\
{$[\mathrm{Ca} / \mathrm{H}]$} & $0.18-0.25$ \\
{$[\mathrm{Ca} / \mathrm{H}]_{\text {median }}$} & 0.21
\end{tabular}

where $F_{\mathrm{X}}$ is the $\mathrm{X}$-ray flux of the star, and $E_{g}=G M / R$ is the gravitational potential energy. The evaporation efficiency, $\eta$, is the fraction of the input stellar energy that is converted to escaping outflow from the planet. It is often assumed to be a constant, but it is more likely that its value varies with the age of the system (Owen \& Wu 2013). The evaporation efficiency $\eta$ has been studied by various authors (e.g., Shematovich et al. 2014; Salz et al. 2016) who demonstrate that values between 0.01 and 0.2 are reasonable for our planet range of interest. In other words, $\eta$ hides the complexity of atmospheric radiative transfer of X-ray photons as well as unknown quantities such as the planetary albedo.

The strongest assumption we make is that mass loss is constant over the planet's lifetime, such that $M_{\mathrm{env}, \text { lost }}=t_{\star} \dot{M}$ $\left(t_{\star}=12.9\right.$ Gyr; Takeda et al. 2007). Thus, Equations (4) and (5) provide the expression

$$
P_{\mathrm{b}}=\frac{\eta L_{\mathrm{X}} t_{\star}}{16 \pi a^{2} R},
$$

with $L_{\mathrm{X}}=4 \pi a^{2} \quad F_{\mathrm{X}}=4 \times 10^{26} \mathrm{erg} \mathrm{s}^{-1}$ (Porto de Mello et al. 2006) being the X-ray luminosity of the star. In Figure 3 , we compute $\Delta R / R$ as a function of $\eta$, since the exact value of $\eta$ is not well known. Fortunately, $\Delta R / R$ depends weakly on $\eta$. Also, uncertainty in stellar age only has a small effect on $\Delta R / R$ : a difference in stellar age of $1 \mathrm{Gyr}$ only introduces variations on $\Delta R / R$ of less than one percent. The spread in $\Delta R / R$ is mainly due to the uncertainties in planetary mass and radius.

The physical interpretation of the preceding expressions for $P_{\mathrm{b}}$ and $\Delta R$ are worth emphasizing. The former is the amount of primordial atmosphere that may be lost by atmospheric escape during the lifetime of the star. It provides a conservative estimate because we have assumed the X-ray luminosity to be constant, whereas in reality stars tend to be brighter in X-rays earlier in their lifetimes:

$$
M_{\mathrm{env}, \mathrm{lost}}=\int_{0}^{t_{\star}} \dot{M}(t) d t>\dot{M} t_{\star} .
$$

The expression for $\Delta R$ is then a lower limit for a primordial atmosphere thickness corresponding to this atmospheric massloss scenario. 


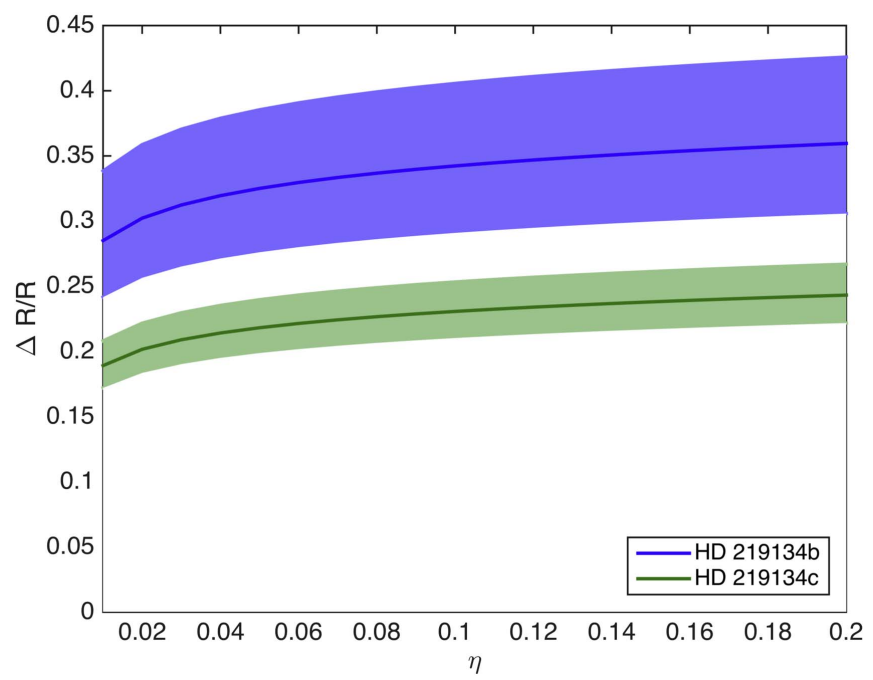

Figure 3. Threshold thickness $\Delta R$ as a function of evaporation efficiency $\eta$. The spread accounts for the uncertainty in planet mass and radius, and age. If the inferred radius $r_{\text {env }}$ is less than $\Delta R$, then the atmosphere is most likely enriched and not dominated by $\mathrm{H}_{2}$.

\subsection{Assessing Secondary/Primordial Nature of an Atmosphere}

We compare $\Delta R$ with the gas thickness $r_{\text {env }}$ inferred from the interior characterization. There are three possible scenarios:

1. $r_{\mathrm{env}}>\Delta R$. Atmospheric escape is not efficient enough in removing a possible primordial atmosphere. This suggests that a large portion of the atmosphere can be primordial. However, a secondary atmosphere is also possible.

2. $r_{\mathrm{env}} \approx \Delta R$. Mass loss can be still ongoing, and no conclusion can be drawn about the nature of the atmosphere.

3. $r_{\text {env }}<\Delta R$. Atmospheric escape should have efficiently removed any primordial $\mathrm{H}_{2}$ atmosphere. If a finite $r_{\mathrm{env}} / R$ is inferred, the atmosphere is likely enriched and thus of secondary origin. Because the calculation of the threshold thickness $\Delta R$ is conservative, this is the only scenario that can be used for a conclusive statement on the atmospheric origin.

This conclusion is illustrated in Figure 4, where the time evolutions of $\mathrm{H}_{2}$-dominated atmosphere thicknesses for HD $219134 \mathrm{~b}$ are shown for $\eta=0.01$. The curves are constructed such that at $t=t_{\star}$, the relative thicknesses $r_{\text {env }} / R$ are equal to 0 (blue), 0.1 (black), 0.17 (red), and 0.23 (green). Furthermore, the solid curves include the time-evolution of X-ray flux, which we assume here to be solar-like $\left(F_{\mathrm{X}} \propto t^{-1.83}\right.$ for $t>t_{\mathrm{sat}}$ and $F_{\mathrm{X}}=F_{\mathrm{X}}$ for $t<t_{\text {sat }}$, where the saturation time is equal to $100 \mathrm{Myr}$ and $F_{\mathrm{X}}\left(t_{\star}\right)$ is the observed value) (Ribas et al. 2005). Compared with a constant X-ray flux, the higher stellar activity for a young star implies that an atmosphere thickness of $r_{\text {env }} / R$ at $t_{\star}$ must have started with a higher gas fraction at $t=0$ (see the difference between solid and dashed curves in Figure 4). In both scenarios, we find that the smaller the observed thickness $r_{\text {env }} / R$ compared with $\Delta R / R$, the shorter the time a planet spends with this atmosphere thickness. Thus, it is possible for a planet to host remaining small amounts of an initially thick primordial atmosphere that will have a $r_{\text {env }} / R$ lower than the threshold thickness. However, we find that this state is a

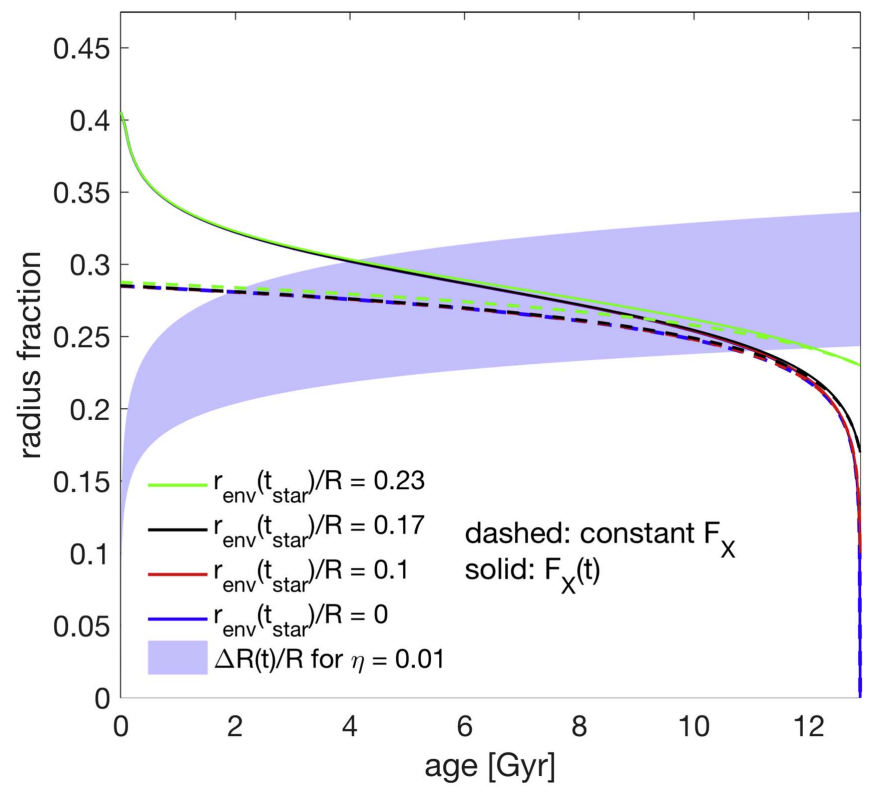

Figure 4. Evolution of $\mathrm{H}_{2}$-dominated atmosphere thicknesses $r_{\mathrm{env}} / R$ for HD $219134 \mathrm{~b}$ leading to different thicknesses at $t=t_{\star}(\eta=0.01$ in all cases $)$. Solid curves account for a time variable stellar X-ray flux $F_{X}(t)$ (Ribas et al. 2005), whereas dashed curves imply a constant $F_{\mathrm{X}}$. The blue-shaded area depicts the evolution of $\Delta R / R$, its spread accounts for the uncertainties in planet mass and radius.

Table 4

Considered Planet Bulk Abundance Cases

\begin{tabular}{lccc}
\hline \hline Parameter & V0 & V1 & V2 \\
\hline $\mathrm{Fe} / \mathrm{Si}_{\text {bulk }}$ & $1.73 \pm 1.55$ & $10.68 \pm 1.55$ & $1.00 \pm 1.55$ \\
$\mathrm{Mg} / \mathrm{Si}_{\text {bulk }}$ & $1.44 \pm 0.91$ & $1.02 \pm 0.91$ & $1.14 \pm 0.91$ \\
$\mathrm{Na}_{2} \mathrm{O}[\mathrm{wt} \%]$ & 0.021 & 0.01 & 0.025 \\
$\mathrm{Al}_{2} \mathrm{O}_{3}[\mathrm{wt} \%]$ & 0.055 & 0.023 & 0.057 \\
$\mathrm{CaO}[\mathrm{wt} \%]$ & 0.021 & 0.01 & 0.021 \\
\hline
\end{tabular}

Note. V0 represents median abundance estimates, whereas V1 and V2 refer to iron-rich and iron-poor cases, respectively.

very short fraction of the planet's lifetime, so it is unlikely that the planets we observe with $r_{\text {env }} / R$ lower than the threshold value will be remnants of thicker primordial atmospheres. Consequently, inferred atmospheres with thicknesses less than $\Delta R / R$ are likely to be enriched (secondary).

\section{Results}

\subsection{Interiors of HD $219134 b$ and $c$}

We apply the inference method to HD $219134 \mathrm{~b}$ and $\mathrm{c}$ with the data listed in Tables 2 and 3. The latter lists different stellar abundance estimates from the literature (Thevenin 1998; Thévenin \& Idiart 1999; Valenti \& Fischer 2005; Ramírez et al. 2007; Mishenina et al. 2015) that were compiled by Dorn et al. (2016a) to examine different bulk abundance scenarios. Besides a median abundance estimate (V0), they provide an iron-rich (V1) and an iron-poor (V2) scenario that reflects the limited accuracy in stellar abundance estimates (Table 4). First, we use the median stellar abundance estimate denoted with V0. Figures 5 and 6 show the 2D and 1D marginal posteriors for all eight model parameters. Best-constrained parameters are the 


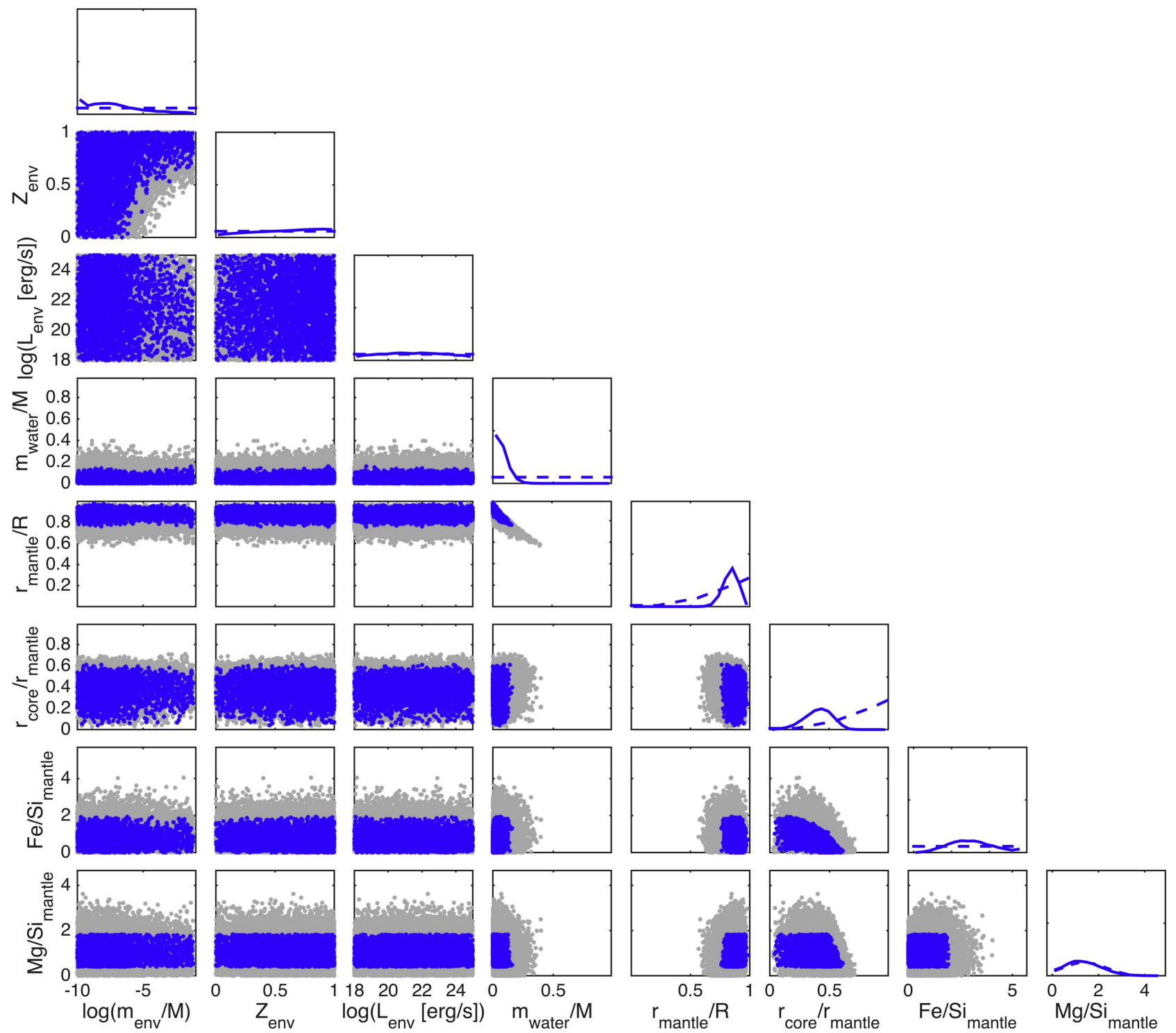

Figure 5. Sampled 2D and 1D marginal posterior for HD $219134 \mathrm{~b}$ interior parameters: gas mass $m_{\text {env }}$, gas metallicity $Z_{\text {env }}$, intrinsic luminosity $L_{\text {env }}$, mass of water $m_{\text {water }}$, radius of rocky interior $r_{\text {mantle }}$, core radius $r_{\text {core }}$, and mantle's relative abundances $\mathrm{Fe} / \mathrm{Si}_{\text {mantle }}$ and $\mathrm{Mg} / \mathrm{Si}$ mantle. Blue dots explain the data within $1 \sigma$ uncertainty. Dashed curves represent the prior distributions assumed.

layer thicknesses represented by $m_{\text {water }}, r_{\text {mantle, }}$ and $r_{\text {core }}$ We summarize our findings on the interiors of HD $219134 \mathrm{~b}$ and $\mathrm{c}$ with respect to the models that fit the data within $1 \sigma$ uncertainty (blue dots in Figures 5 and 6):

1. The possible interiors of HD $219134 \mathrm{~b}$ and c span a large region, including purely rocky and volatile-rich scenarios.

2. Less than $0.1 \%$ of the model solutions for planets $\mathrm{b}$ and $\mathrm{c}$, respectively, are rocky ( $\left.r_{\text {rocks }} / R>0.98\right)$.

3. The possible water mass fraction of HD $219134 \mathrm{~b}$ and $\mathrm{c}$ can reach from $0-0.2$ and $0-0.1$, respectively.

4. Unsurprisingly, the individual atmosphere properties ( $m_{\mathrm{env}}, L_{\mathrm{env}}, Z_{\mathrm{env}}$ ) are weakly constrained. Consequently, their probability distribution functions are dominated by prior information. However, the possible range of atmosphere thickness is well constrained to $0-0.18$ and 0-0.13 for planets $\mathrm{b}$ and $\mathrm{c}$, respectively (see Section 3.1.1).

\subsubsection{Influence of Stellar Abundances}

Dorn et al. (2016a) investigated the influence of different bulk abundance constraints on interior estimates. In Figures 7 and 8 , we similarly show this influence on key interior parameters $\left(r_{\text {env }} / R, m_{\text {water }}, r_{\text {mantle }}\right.$, and $\left.r_{\text {core }}\right)$ for the median abundance estimate (V0, blue), the iron-rich case (V1, light green), and the iron-poor case (V2, dark green) (Table 4). As discussed by Dorn et al. (2016a), the largest effects are seen on $r_{\text {mantle }}$ and $r_{\text {core }}$ if the planets are iron-rich, the core size is significantly larger, which implies a smaller rocky interior ( $\left.r_{\text {mantle }}\right)$ to fit mass. The effect on $r_{\text {env }} / R$ is apparent in the comparison between the iron-rich case V1 and V0. For an ironrich planet, the density of the rocky interior is higher. To fit the mass, $r_{\text {mantle }}$ is smaller. Consequently, to fit the radius, $r_{\mathrm{env}} / R$ can be larger. For the iron-rich case (V1), the upper $99 \%$ percentile of $r_{\text {env }} / R$ is 0.02 (HD $219134 \mathrm{~b}$ ) and 0.04 (HD 219134 c) larger than for V0. Even if the iron-rich case 


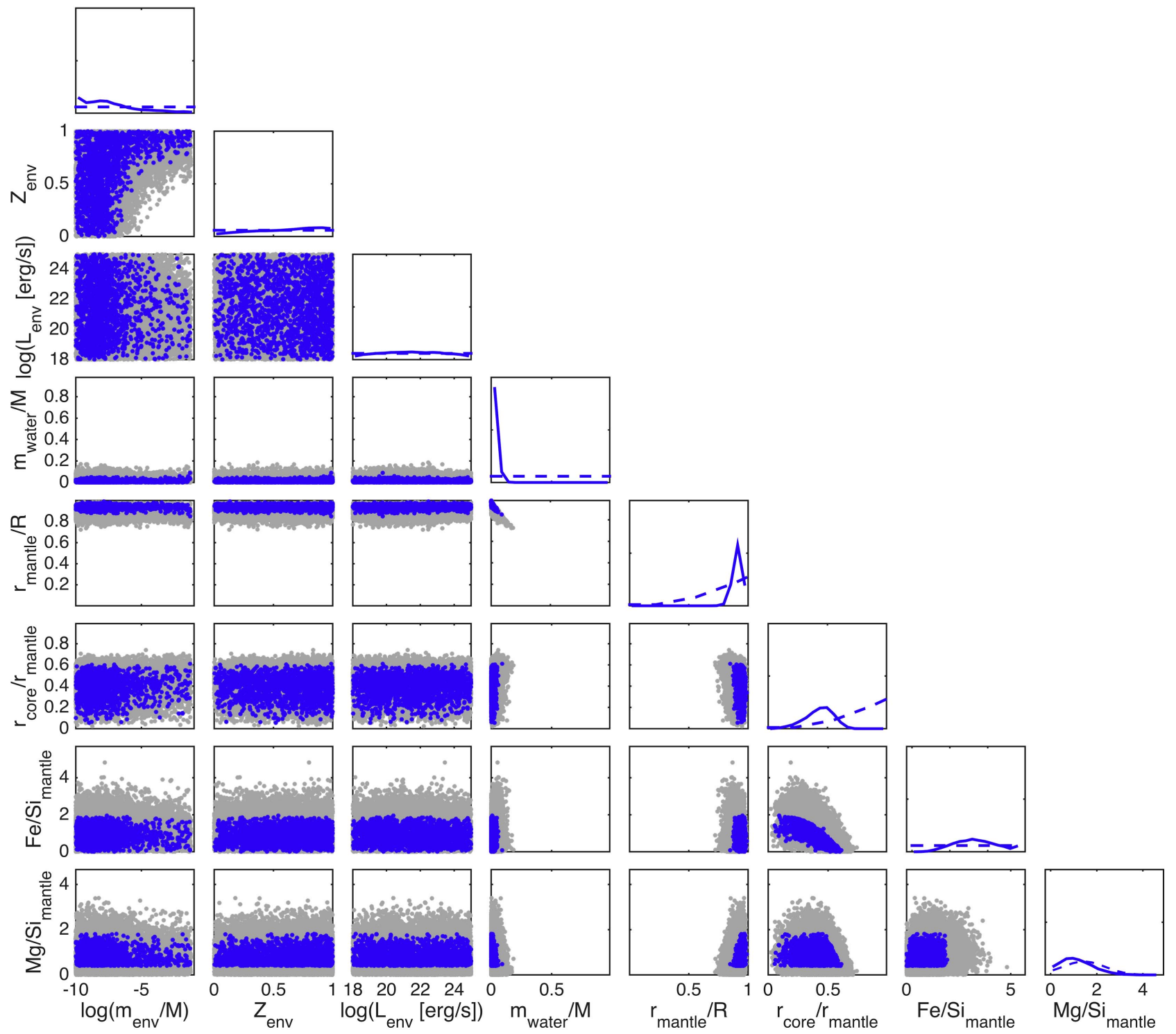

Figure 6. Sampled 2D and 1D marginal posterior for HD $219134 \mathrm{c}$ interior parameters: gas mass $m_{\text {env }}$, gas metallicity $Z_{\text {env }}$, intrinsic luminosity $L_{\text {env }}$, mass of water $m_{\text {water }}$, radius of rocky interior $r_{\text {mantle }}$, core radius $r_{\text {core }}$, and mantle's relative abundances $\mathrm{Fe} / \mathrm{Si}_{\text {mantle }}$ and $\mathrm{Mg} / \mathrm{Si}$ mantle. Blue dots explain the data within $1 \sigma$ uncertainty. Dashed curves represent the prior distributions assumed.

is in agreement with spectroscopic data, we believe that V1 may represent a limitation in accuracy rather than the actual planet bulk abundance.

\subsubsection{Influence of Data Uncertainty}

In Figures 7 and 8, we also investigate the improvement in constraining interior parameters assuming the hypothetical case of having double the precision on (1) observed mass (light purple), and (2) mass and radius (dark purple). Significant improvement in constraining interior parameters is only obvious for HD $219134 \mathrm{~b}$ when both mass and radius precision is doubled. For HD $219134 \mathrm{c}$, the increase in both mass and radius uncertainty leads to only moderate improvement of parameter estimates. The different potential to improve interior estimates by reducing data uncertainty for planets $\mathrm{b}$ and $\mathrm{c}$ stems from the fact that the uncertainties are much smaller for planet $\mathrm{c}$ $\left(\sigma_{R}=3.1 \%\right)$ compared with b $\left(\sigma_{R}=5.4 \%\right)$. For the considered planets, improved constraints for interior parameters are dominantly gained by a better precision in radius. This is expected, since mass-radius curves flatten out at higher masses (Figure 1).

\subsubsection{Influence of Prior on $Z_{e n v}$}

We have shown that the individual parameters $L_{\mathrm{env}}, Z_{\mathrm{env}}$, and $m_{\text {env }}$ are weakly constrained and are therefore dominated by their prior distributions. However, $r_{\text {env }} / R$ is well constrained (Figures 7 and 8), which is not explicitly a model parameter in this study. Here, we investigate the effect of different priors on the radius fractions $r_{\text {env }} / R$. An obvious prior to test is on $Z_{\text {env }}$. The prior on gas metallicity can be chosen such that it favors $\mathrm{H}_{2}$-dominated (uniform in $1 / Z_{\text {env }}$ ) or enriched atmospheres (uniform in $Z_{\text {env }}$ ). In Figures 7 and 8 (comparing blue and dark gray curve), we demonstrate that different priors in $Z_{\text {env }}$ have only small effects on the possible distribution of radius fractions $r_{\text {env }} / R$. 

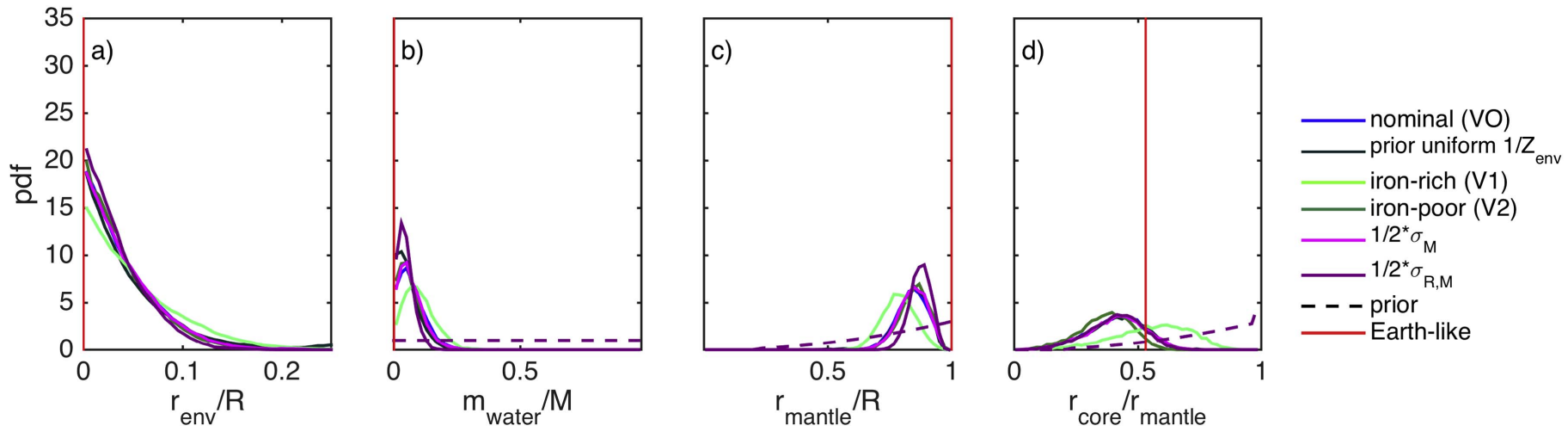

Figure 7. Sampled 1D marginal posterior for selected parameters of HD $219134 \mathrm{~b}$ : (a) gas radius fraction $r_{\text {env }} / R$, (b) water mass fraction $m_{\text {water }} / M$, (c) rock radius

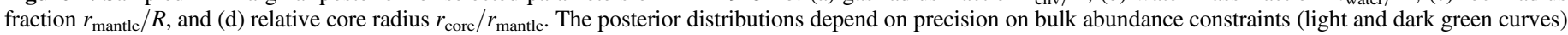
and mass and radius uncertainties (light and dark purple curves). For comparison, the Earth-like solution is highlighted in red.

\subsection{Secondary or Primordial Atmosphere?}

The comparison in Figure 9 of the inferred $r_{\text {env }} / R$ (solid lines) with the threshold thickness $\Delta R / R$ (dashed areas) shows that the possible atmospheres of planets $\mathrm{b}$ and $\mathrm{c}$ are significantly smaller than $\Delta R / R$. This indicates that the possible atmospheres are not dominated by hydrogen, but must be secondary in nature. This provides a simple test to identify $\mathrm{H}_{2}$-rich versus enriched atmospheres, which may then guide future spectroscopic campaigns to characterize atmospheres (e.g., JWST, E-ELT).

\subsection{Comparison with Other Planets}

Similar to HD $219134 \mathrm{~b}$ and $\mathrm{c}$, we compare $r_{\text {env }} / R$ with $\Delta R / R$ (Figure 9 and Table 5) for GJ $1214 \mathrm{~b}$, HD $97658 \mathrm{~b}$, and $55 \mathrm{Cnc}$ e. This serves as a benchmark for our proposed determination for $\mathrm{H}_{2}$-dominated and enriched atmospheres, as large efforts were put in understanding composition and nature of the atmospheres of the three planets.

For GJ $1214 \mathrm{~b}$, the distribution of $r_{\text {env }} / R$ is large and overlaps with $\Delta R / R$. The possible atmosphere is consistent with both a $\mathrm{H}_{2}$-dominated and enriched atmosphere. Prior to our study, much effort has been invested in characterizing the atmosphere of GJ 1214 b (e.g., Berta et al. 2012; Kreidberg et al. 2014). Studies on interior structure suggested either an hydrogen-rich atmosphere that formed by recent outgassing or a maintained hydrogen-helium atmosphere of primordial nature (Rogers \& Seager 2010). A third scenario of a massive water layer surrounded by a dense water atmosphere has been disfavored by Nettelmann et al. (2011) based on thermal evolution calculations that argued that the water-to-rock ratios would be unreasonable large. Transmission spectroscopy and photometric transit observations revealed that the atmosphere has clouds and/or shows features from a high mean-molecularmass composition (Berta et al. 2012; Kreidberg et al. 2014).

For HD $97658 \mathrm{~b}$, we find that $r_{\text {env }} / R$ is very likely smaller than $\Delta R / R$. This suggests an atmosphere that is enriched and thus possibly of secondary nature; however, a primordial atmosphere cannot be ruled out with certainty. Previous transmission spectroscopy results of Knutson et al. (2014) are in agreement with a flat transmission spectrum, indicating either a cloudy or water-rich atmosphere. The latter scenario would involve photodissociation of water into $\mathrm{OH}$ and $\mathrm{H}$ at high altitudes. Evidence for this would be neutral hydrogen escape. Bourrier et al. (2016) undertook a dedicated Ly $\alpha$ line search of three transits but could not find any signature. Any neutral hydrogen escape could happen at low rates only. Consequently, a low hydrogen content in the upper atmosphere is a likely scenario. This is consistent with our findings, that a secondary atmosphere is probable.

For $55 \mathrm{Cnc}$ e, our prediction clearly indicates a secondary atmosphere, as $r_{\text {env }} / R$ is significantly lower than $\Delta R / R$. This is in agreement with previous interpretations based on infrared and optical observations of transits, occultations, and phase curves (Demory et al. 2012, 2016; Angelo \& Hu 2017). This planet has a large day-night-side temperature contrast of about $1300 \mathrm{~K}$, and its hottest spot is shifted eastwards from the substellar point (Demory et al. 2016; Angelo \& Hu 2017). The implication for the atmosphere is an optically thick atmosphere with inefficient heat redistribution. A bare rocky planet is disfavored (Angelo \& Hu 2017). Furthermore, Ehrenreich et al. (2012) give evidence for no extended hydrogen planetary atmospheres (but see Tsiaras et al. 2016). If an atmosphere is present, it would be of secondary nature. Our approximated approach leads to the same conclusion. Furthermore, the study of $55 \mathrm{Cnc}$ e's thermal evolution and atmospheric evaporation by Lopez (2017) suggest either a bare rocky planet or a waterrich interior. Although the composition of $55 \mathrm{Cnc} e$ is a matter of debate, a hydrogen-dominated atmosphere seems unlikely.

We also note that this test holds for Earth and Venus, although atmospheric loss mechanisms are very different for them (i.e., Jeans escape and non-thermal escape) (Shizgal \& Arkos 1996). The threshold thicknesses of possible primordial atmospheres are larger than $10 \%$, whereas the actual thicknesses are no more than a few percent. Thus, our tests would correctly predict a secondary atmosphere for Earth and Venus.

A comparison of atmospheric origin on a larger set of exoplanets is limited due to the lack of estimated X-ray stellar luminosities. For simplicity, we assume solar X-ray luminosities whenever stellar X-ray luminosities are not available, which is a fair assumption given that the Kepler mission targeted Sun-like stars. Using such simple assumptions, the distribution of planets with secondary atmospheres depends on planet mass and equilibrium temperature to first order (Figure 10 and Table 6). In comparison with the tested HD $219134 \mathrm{~b}$ and c, most planets have higher equilibrium temperatures and are thus more vulnerable against atmospheric loss. Also, Dorn et al. (2016a) concluded that it is unlikely that the planets HD 219134 b, Kepler-10 b, Kepler-93 b, CoRoT-7b, 

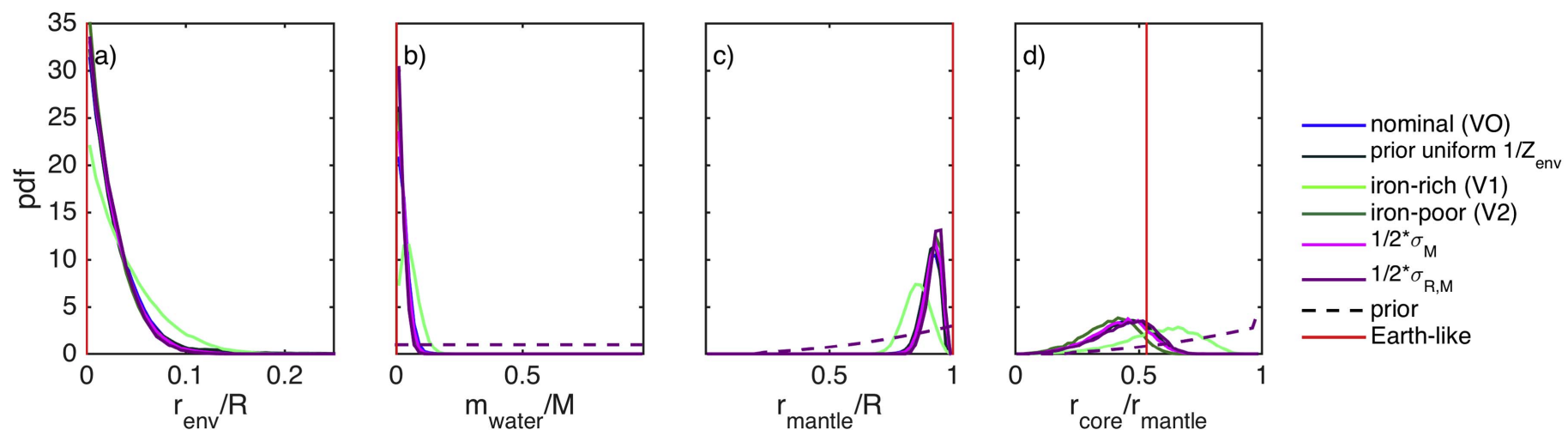

Figure 8. Sampled 1D marginal posterior for selected parameters of HD $219134 \mathrm{c}$ : (a) gas radius fraction $r_{\text {env }} / R$, (b) water mass fraction $m_{\text {water }} / M$, (c) rock radius fraction $r_{\text {mantle }} / R$, and (d) relative core radius $r_{\text {core }} / r_{\text {mantle. }}$. The posterior distributions depend on precision on bulk abundance constraints (light and dark green curves) and mass and radius uncertainties (light and dark purple curves). For comparison, the Earth-like solution is highlighted in red.

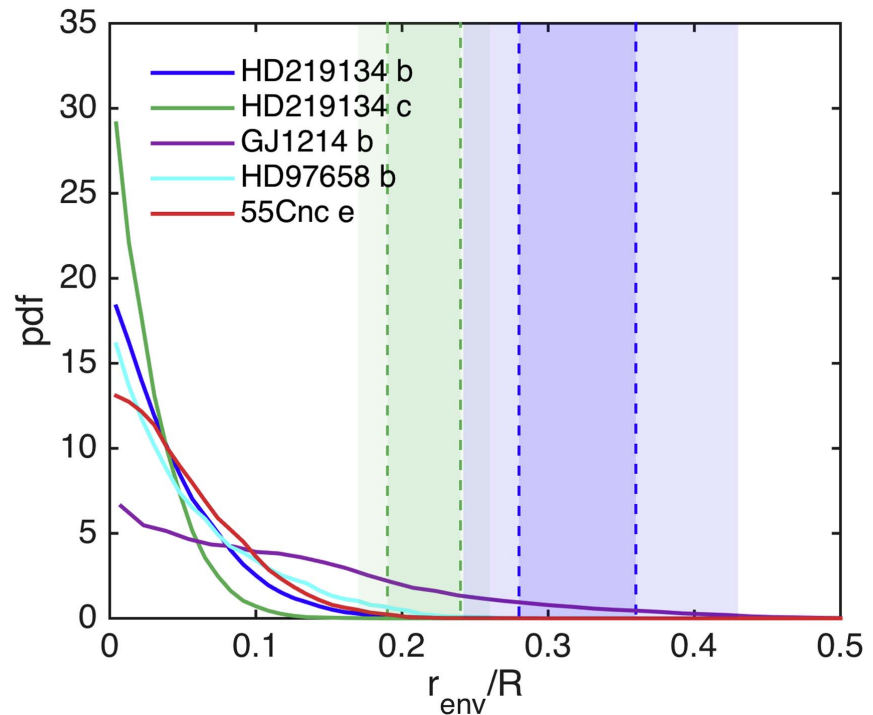

Figure 9. Comparison of $r_{\text {env }} / R$ between five highlighted planets in Figure 1. Inferred radius (solid lines) and approximated threshold thicknesses $\Delta R / R$ (colored areas with dashed borders). $\Delta R / R$ is listed in Table 5 for all five planets.

Table 5

Threshold Thickness $\Delta R / R$ for Different Evaporation Efficiencies $\eta$

\begin{tabular}{lrcc}
\hline \hline Planet & $L_{\mathrm{x}}\left(\mathrm{erg} \mathrm{s}^{-1}\right)$ & $\begin{array}{c}\Delta R / R \\
(\eta=0.01)\end{array}$ & $\begin{array}{c}\Delta R / R \\
(\eta=0.2)\end{array}$ \\
\hline HD 219134 b & $4 \times 10^{26}$ & 0.28 & 0.36 \\
HD 219134 c & $4 \times 10^{26}$ & 0.19 & 0.24 \\
GJ 1214 b & $7.4 \times 10^{25}$ & 0.17 & 0.22 \\
55 Cnc e & $4 \times 10^{26}$ & 0.37 & 0.46 \\
HD 97658 b & $1.2 \times 10^{28}$ & 0.18 & 0.22 \\
Earth & $2.24 \times 10^{27}$ & 0.12 & 0.16 \\
Venus & $2.24 \times 10^{27}$ & 0.15 & 0.21 \\
\hline
\end{tabular}

and 55 Cnc e could retain a hydrogen-dominated atmosphere against evaporative mass loss.

The possible transition between secondary and primordial atmospheres depending on $T_{\mathrm{eq}}$ is positively correlated with planet mass (Figure 10). Theoretical photoevaporation studies (e.g., Lopez \& Fortney 2013; Jin et al. 2014) and the study on observed planets by Lecavelier des Etangs (2007) predict similar trends, in that planets need to be more massive when receiving higher incident flux in order to retain their primordial

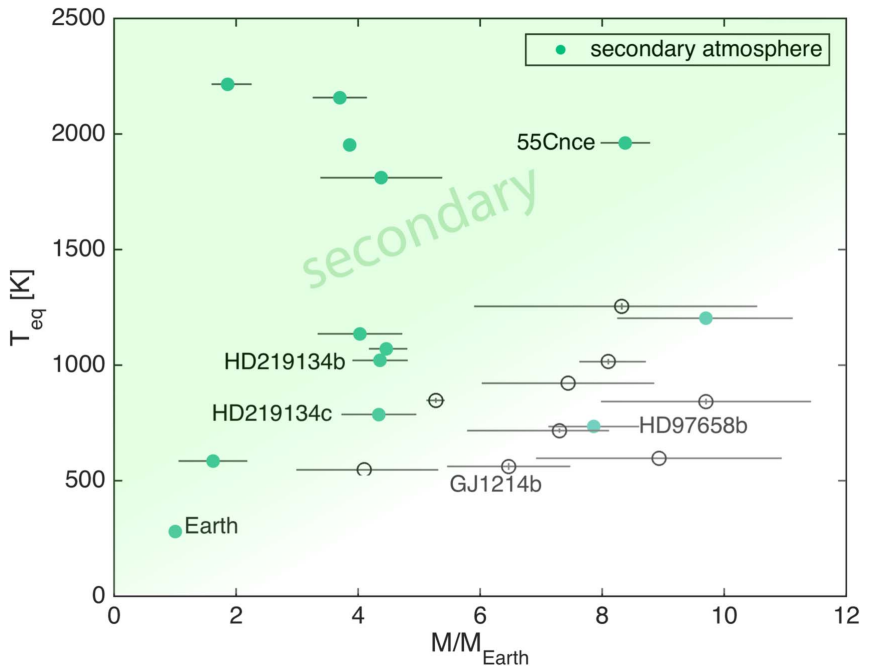

Figure 10. Possible origin of atmospheres depending on effective temperature and planet mass. For labeled planets, we use our method described in the text. For unlabeled planets, stellar X-ray luminosities are not available, thus we assume solar X-ray luminosities which is a fair assumption given that the Kepler mission targeted Sun-like stars. Radii and masses of considered planets are shown in Figure 1.

atmospheres. For a better understanding of the observed distribution of secondary atmospheres, future estimates of $\mathrm{X}$-ray stellar luminosities are required.

\section{Discussion}

As already mentioned, the strongest assumption we make is that mass loss is constant over the stellar age. A more accurate approach is to calculate

$$
M_{\mathrm{env}, \mathrm{lost}}=\int_{0}^{t_{\star}} \frac{\pi \eta F_{\mathrm{X}} R^{2}}{E_{g}} d t
$$

where $\eta(t)$ and $F_{\mathbf{X}}(t)$ are both functions of time. The X-ray luminosity evolves over the lifetime of the star, which in turn causes the efficiency of atmospheric escape to evolve. Also, the planetary radius $R$ depends on the gas mass fraction that changes over time. We emphasize that, while the $M_{\text {env, lost }}=\dot{M} t_{\star}$ approximation may lack precision, the logical structure of our approach is robust and accurate. The reasoning remains that $M_{\text {env,lost }}$ (corresponding to a thickness of $\Delta R$ ) worth of atmosphere may be eroded over the stellar lifetime, so 
Table 6

Threshold Thickness $\Delta R / R$ for Evaporation Efficiencies $\eta$ of 0.01 and 95th Percentile of Inferred Atmosphere Thicknesses $r_{\text {env }} / R$

\begin{tabular}{lcc}
\hline \hline Planet & 95th Percentile of $r_{\text {env }} / R$ & $\Delta R / R$ \\
\hline Kepler-78 b & 0.15 & $1.0^{\mathrm{a}}$ \\
GJ 1132 b & 0.1 & $0.40^{\mathrm{a}}$ \\
Kepler-93 b & $<0.05$ & $0.31^{\mathrm{a}}$ \\
Kepler-10 b & $<0.05$ & $0.76^{\mathrm{a}}$ \\
Kepler-36 b & $<0.05$ & $0.23^{\mathrm{a}}$ \\
HD 219134 c & 0.13 & 0.19 \\
HD 219134 b & 0.18 & 0.28 \\
CoRoT-7 b & 0.1 & $0.59^{\mathrm{a}}$ \\
Kepler-21 b & 0.05 & $0.64^{\mathrm{a}}$ \\
Kepler-20 b & 0.05 & $0.18^{\mathrm{a}}$ \\
55 Cnc e & 0.18 & 0.37 \\
Kepler-19 b & 0.27 & $0.25^{\mathrm{a}}$ \\
Kepler-102 e & 0.17 & $0.10^{\mathrm{a}}$ \\
HD 97658 b & 0.21 & 0.18 \\
Kepler-68 b & 0.28 & $0.25^{\mathrm{a}}$ \\
Kepler-454 b & 0.28 & $0.16^{\mathrm{a}}$ \\
GJ 1214 b & 0.39 & 0.17 \\
Kepler-11 d & 0.43 & $0.20^{\mathrm{a}}$ \\
Kepler-33 c & 0.48 & $0.18^{\mathrm{a}}$ \\
Kepler-79 e & 0.58 & $0.25^{\mathrm{a}}$ \\
Kepler-36 c & 0.49 & $0.30^{\mathrm{a}}$ \\
\hline
\end{tabular}

Note.

${ }^{a}$ In cases of planets for which stellar X-ray luminosities are not available, we assume solar X-ray luminosities.

any inferred atmosphere with thicknesses less than this threshold are very unlikely to be primordial $\left(\mathrm{H}_{2}\right.$-dominated).

In addition, we assume $T=T_{\text {eq }}$ while estimating the threshold thickness (Equation (3)). Heng (2016) finds differences on the order of few tens of percents while approximating the scale height with the isothermal scale height at $T=T_{\text {eq. }}$. If temperatures are higher, the hydrogen escape would be more efficient and $\Delta R / R$ would be higher (and vice versa). The uncertainty on the temperature is accounted for by the variability in $\eta$.

Furthermore, our estimates of the radius fraction $r_{\mathrm{env}} / R$ are subject to our choices of interior model and assumptions. Changes in the interior model, especially the atmosphere model, can affect the estimated $r_{\text {env }} / R$ as discussed by Dorn et al. (2016b). Furthermore, we assume distinct layers of core, mantle, water, and gas. This may not be true as discussed for giant planets (Stevenson 1985; Helled et al. 2010).

Following the outlined strategy, it is possible to test for other types of atmospheres (e.g., $\mathrm{N}_{2}$ or $\mathrm{CO}_{2}$-dominated atmospheres). Here, we focused on an atmosphere type that informs us about formation processes, i.e., we have assumed that a primordial atmosphere is dominated by hydrogen. In principle, a primordial atmosphere can be enriched by planetesimal disruption during the accretion. However, initial gas fractions for super-Earths are small, and it is not clear whether atmosphere enrichment can be efficient in these cases nor if metal-enriched thin atmospheres remain well mixed over long timescales.

We have demonstrated that the possible atmospheres on HD $219134 \mathrm{~b}$ and c are very likely to be secondary in nature. We have shown that this result is robust against different assumptions of bulk abundance constraints and prior choices, as shown for $Z_{\text {env }}$.
Based on bulk density, both planets could be potentially rocky. However, we would expect planets, that are rocky and that formed within the same disk, to roughly lie on the same mass-radius curve. This is because we expect a compositional correlation, i.e., similar abundances of relative refractory elements (e.g., Sotin et al. 2007). The fact that HD 219134 b and $\mathrm{c}$ do not fall on one mass-radius curve, suggests that the larger planet $b$ must harbor a substantial volatile layer.

Our use of stellar composition as a proxy for the planet bulk composition excludes Mercury-like rocky interiors. If such interiors were applicable to the HD 219134 planets, the rocky interiors would be iron-rich surrounded by substantially thick volatile envelopes in order to fit mass and radius. It remains an open question whether Mercury-like interiors are common.

\section{Conclusions and Outlook}

We have presented a method to determine the nature of a possible atmosphere. As close-in planets suffer from evaporative mass loss, the amount of primordial atmosphere that can be lost is determined by irradiation from the star, lifetime of the system, and evaporation efficiency. Fortunately, the amount of primordial atmosphere loss is weakly dependent on evaporation efficiency and system lifetime in case of the usually Gyr-old observed exoplanets. A comparison between the threshold thickness above which a primordial atmosphere can be retained against atmospheric escape and the actual possible atmosphere thickness is a clear indicator of whether an atmosphere is secondary. We performed this analysis for HD $219134 \mathrm{~b}$ and HD $219134 \mathrm{c}$.

The possible thicknesses of their atmospheres were inferred by using a generalized Bayesian inference method. For this, we have used the data of planet mass, radius, stellar irradiation, and bulk abundance constraints from the star to constrain the interiors of HD $219134 \mathrm{~b}$ and c. Interior parameters include core size; mantle composition and size; water mass fraction; intrinsic luminosity; gas mass; and gas metallicity. Although individual parameters of the gas layer $\left(m_{\mathrm{env}}, L_{\mathrm{env}}, Z_{\mathrm{env}}\right)$ are only weakly constrained, the thickness is well contrained. Inferred thicknesses $r_{\text {env }} / R$ are robust against different assumed priors and bulk abundance constraints.

We summarize our findings on HD $219134 \mathrm{~b}$ and HD 219134 c below:

1. Maximum radius fractions of possible gas layers are 0.18 (HD 219134 b) and 0.13 (HD 219134 c),

2. Possible atmospheres are likely secondary in nature,

3. HD $219134 \mathrm{~b}$ must contain a significant amount of volatiles.

Here, we have proposed a simple quantitative determination of the nature of an exoplanetary atmosphere, that does not include spectroscopic measurement. To check our method against planets whose atmospheres are intensively studied, we applied it to GJ 1214 b, HD 97658 b, and 55 Cnc e. Our predictions agree with previous findings on their atmospheres and may be tested by future infrared transmission spectroscopy performed on these exoplanets.

We thank Yann Alibert for constructive comments. This work was supported by the Swiss National Foundation under grant 15-144. It was in part carried out within the frame of the National Centre for Competence in Research PlanetS. 


\section{ORCID iDs}

Kevin Heng (1) https://orcid.org/0000-0003-1907-5910

\section{References}

Adams, E. R., Seager, S., \& Elkins-Tanton, L. 2008, ApJ, 673, 1160 Angelo, I., \& Hu, R. 2017, arXiv:1710.03342

Berta, Z. K., Charbonneau, D., Désert, J. M., et al. 2012, ApJ, 747, 35

Bouchet, J., Mazevet, S., Morard, G., Guyot, F., \& Musella, R. 2013, PhRvB, 87, 094102

Bourrier, V., Ehrenreich, D., King, G., et al. 2016, A\&A, 597, A26

Connolly, J. A. D. 2009, GGG, 10

Demory, B. O., Gillon, M., De Wit, J., et al. 2016, Natur, 532, 207

Demory, B. O., Gillon, M., Seager, S., et al. 2012, ApJL, 751, L28

Dorn, C., Hinkel, N. R., \& Venturini, J. 2016a, A\&A, 597, A38

Dorn, C., Khan, A., Heng, K., et al. 2015, A\&A, 577, A83

Dorn, C., Venturini, J., Khan, A., et al. 2016b, A\&A, 597, A37

Dressing, C. D., \& Charbonneau, D. 2015, ApJ, 807, 45

Dziewonski, A. M., \& Anderson, D. L. 1981, PEPI, 25, 297

Ehrenreich, D., Bourrier, V., Bonfils, X., et al. 2012, A\&A, 547, A18

Ehrenreich, D., \& Desert, J. M. 2011, A\&A, 529, A136

Elkins-Tanton, L. T., \& Seager, S. 2008, ApJ, 685, 1237

Fortney, J. J., Mordasini, C., Nettelmann, N., et al. 2013, ApJ, 775, 80

Freedman, R. S., Lustig-Yaeger, J., Fortney, J. J., et al. 2014, ApJS, 214, 25

Gordon, S., \& McBride, B. 1994, Computer Program for Calculation of

Complex Chemical Equilibrium Compositions and Applications (Cleveland,

$\mathrm{OH}$; Springfield, VA: National Aeronautics and Space Administration,

Office of Management, Scientific and Technical Information Program; National Technical Information Service, Distributor)

Gillon, M., Demory, B.-O., Van Grootel, V., et al. 2017, NatAs, 1, 0056

Guillot, T. 2010, A\&A, 520, A27

Hashimoto, G. L., Abe, Y., \& Sugita, S. 2007, JGRE, 112

Helled, R., Anderson, J. D., Podolak, M., \& Schubert, G. 2010, ApJ, 726, 15

Heng, K. 2016, ApJL, 826, L16

Heng, K., Mendonça, J. M., \& Lee, J. M. 2014, ApJS, 215, 4

Hinkel, N. R., Timmes, F. X., Young, P. A., Pagano, M. D., \& Turnbull, M. C. 2014, AJ, 148, 54

Hu, R., Seager, S., \& Yung, Y. L. 2015, ApJ, 807, 8

Jin, S., Mordasini, C., Parmentier, V., et al. 2014, ApJ, 795, 65

Knutson, H. A., Dragomir, D., Kreidberg, L., et al. 2014, ApJ, 794, 155
Kreidberg, L., Bean, J. L., Désert, J. M., et al. 2014, Natur, 505, 69

Lammer, H., Erkaev, N. V., Odert, P., et al. 2013, MNRAS, 430, 1247

Lecavelier des Etangs, A. 2007, A\&A, 461, 1185

Lecavelier des Etangs, A., Pont, F., Vidal-Madjar, A., \& Sing, D. 2008, A\&A, 481, L83

Leconte, J., Forget, F., \& Lammer, H. 2015, ExA, 40, 449

Lopez, E. D. 2017, MNRAS, 472, 245

Lopez, E. D., \& Fortney, J. J. 2013, ApJ, 776, 2

Mihalas, D. 1970, Stellar Atmospheres (1st ed.; San Francisco: Freeman)

Miller-Ricci, E., Seager, S., \& Sasselov, D. 2009, ApJ, 690, 1056

Mishenina, T., Gorbaneva, T., Pignatari, M., Thielemann, F.-K., \& Korotin, S. A. 2015, MNRAS, 454, 1585

Motalebi, F., Udry, S., Gillon, M., et al. 2015, A\&A, 584, A72

Nettelmann, N., Fortney, J. J., Kramm, U., \& Redmer, R. 2011, ApJ, 733, 2

Owen, J. E., \& Jackson, A. P. 2012, MNRAS, 425, 2931

Owen, J. E., \& Wu, Y. 2013, ApJ, 775, 105

Porto de Mello, G., del Peloso, E. F., \& Ghezzi, L. 2006, AsBio, 6, 308

Ramírez, I., Prieto, C. A., \& Lambert, D. L. 2007, A\&A, 465, 271

Ribas, I., Guinan, E. F., Guedel, M., \& Audard, M. 2005, ApJ, 622, 680

Rogers, L. A. 2015, ApJ, 801, 41

Rogers, L. A., \& Seager, S. 2010, ApJ, 716, 1208

Salz, M., Schneider, P. C., Czesla, S., \& Schmitt, J. H. M. M. 2016, A\&A, $585, \mathrm{~L} 2$

Schaefer, L., \& Fegley, B. 2007, Icar, 186, 462

Seager, S., Kuchner, M., Hier-Majumder, C. A., \& Militzer, B. 2007, ApJ, 669,1279

Shematovich, V. I., Ionov, D. E., \& Lammer, H. 2014, A\&A, 571, A94

Shizgal, B. D., \& Arkos, G. G. 1996, RvGeo, 34, 483

Sotin, C., Grasset, O., \& Mocquet, A. 2007, Icar, 191, 337

Stevenson, D. J. 1985, Icar, 62, 4

Takeda, G., Ford, E. B., Sills, A., et al. 2007, ApJS, 168, 297

Thevenin, F. 1998, yCat

Thévenin, F., \& Idiart, T. P. 1999, ApJ, 521, 753

Thiabaud, A., Marboeuf, U., Alibert, Y., Leya, I., \& Mezger, K. 2015, A\&A, 580, A 30

Tsiaras, A., Rocchetto, M., Waldmann, I. P., et al. 2016, ApJ, 820, 99

Valenti, J. A., \& Fischer, D. A. 2005, ApJS, 159, 141

Vazan, A., Kovetz, A., Podolak, M., \& Helled, R. 2013, MNRAS, stt1248

Venturini, J., Alibert, Y., \& Benz, W. 2016, arXiv:1609.00960

Vogt, S. S., Burt, J., Meschiari, S., et al. 2015, ApJ, 814, 12

Zahnle, K., Schaefer, L., \& Fegley, B. 2010, Cold Spring Harbor Perspectives in Biology, 2, a004895 